\title{
Avatar acceptability: views from the Australian Cystic Fibrosis community on the use of personalised organoid technology to guide treatment decisions
}

\author{
Laura K. Fawcett (10 ${ }^{1,2,3}$, Claire E. Wakefield ${ }^{1,4}$, Sheila Sivam ${ }^{5}$, \\ Peter G. Middleton $\mathbb{1}^{6}$, Peter Wark (10) ${ }^{7}$, John Widger ${ }^{1,2,3}$, Adam Jaffe ${ }^{1,2,3}$ and \\ Shafagh A. Waters $\mathbb{1}^{1,2,3}$
}

Affiliations: 'School of Women's and Children's Health, Faculty of Medicine, University of New South Wales, Sydney, NSW, Australia. ${ }^{2}$ Molecular and Integrative Cystic Fibrosis Research Centre, University of New South Wales, Sydney and Sydney Children's Hospital, Sydney, NSW, Australia. ${ }^{3}$ Dept of Respiratory Medicine, Sydney Children's Hospital, Sydney, NSW, Australia. 'Kids Cancer Centre, Sydney Children's Hospital, Sydney, NSW, Australia. ${ }^{5}$ Dept of Respiratory and Sleep Medicine, Royal Prince Alfred Hospital, Sydney, NSW, Australia. ${ }^{6}$ Cystic Fibrosis Unit, Dept of Respiratory and Sleep Medicine, Westmead Hospital, Sydney, NSW, Australia. ${ }^{7}$ Centre for Healthy Lungs, University of Newcastle, Newcastle, NSW, Australia.

Correspondence: Shafagh A. Waters, 418, 4E, Wallace Wurth Building, UNSW, Sydney, Australia.

E-mail: Shafagh.watersवunsw.edu.au

\section{ABSTRACT}

Background: Patient-oriented research approaches that reflect the needs and priorities of those most affected by health research outcomes improves translation of research findings into practice. Targeted therapies for cystic fibrosis (CF) are now a viable treatment option for some eligible individuals despite the heterogeneous patientspecific therapeutic response. This has necessitated development of a clinical tool that predicts treatment response for individual patients. Patient-derived mini-organs (organoids) have been at the forefront of this development. However, little is known about their acceptability in CF patients and members of the public.

Methods: We used a cross-sectional observational design to conduct an online survey in people with CF, their carers and community comparisons. Acceptability was examined in five domains: 1) willingness to use organoids, 2) perceived advantages and disadvantages of organoids, 3) acceptable out-of-pocket costs, 4) turnaround time and 5) source of tissue.

Results: In total, 188 participants completed the questionnaire, including adults with CF and parents of children with CF (90 (48\%)), and adults without CF and parents of children without CF (98 (52\%)). Use of organoids to guide treatment decisions in CF was acceptable to 86 (95\%) CF participants and 98 (100\%) community participants. The most important advantage was that organoids may improve treatment selection, improving the patient's quality of life and life expectancy. The most important disadvantage was that the organoid recommended treatment might be unavailable or too expensive.

Conclusions: These findings indicate acceptance of patient-derived organoids as a tool to predict treatment response by the majority of people surveyed. This may indicate successful future implementation into healthcare systems.

@ERSpublications

The perspective regarding clinical use of patient-derived organoid models to enable cystic fibrosis personalised therapeutic decision-making of $\mathbf{1 8 8}$ participating adults surveyed https://bit.ly/30nWDJ7

Cite this article as: Fawcett LK, Wakefield CE, Sivam S, et al. Avatar acceptability: views from the Australian Cystic Fibrosis community on the use of personalised organoid technology to guide treatment decisions. ERJ Open Res 2021; 7: 00448-2020 [https://doi.org/10.1183/23120541.00448-2020].

This article has supplementary material available from openres.ersjournals.com

Received: 30 June 2020 | Accepted after revision: 18 Sept 2020

Copyright $\odot$ ERS 2021. This article is open access and distributed under the terms of the Creative Commons Attribution Non-Commercial Licence 4.0. 


\section{Introduction}

Treatment for cystic fibrosis (CF) has developed rapidly over the last decade with the advent of targeted therapies. CF transmembrane conductance regulator (CFTR) modulator therapies [1-3] target the underlying cause of the disease, the CFTR protein. An absent or dysfunctional CFTR protein results in the clinical phenotype of cystic fibrosis [2, 4]. Over 2000 genetic variants of CFTR have been documented, with 322 of these known to result in disease pathology [5]. Amongst individuals with CF, heterogeneity of clinical presentations is broad [6] and is not explained by their CFTR mutation alone. Patients with the same CFTR mutation can show dramatic variation in the severity of organ dysfunction [6]. In addition, response to treatment is known to be heterogeneous with some individuals experiencing improvements, whilst others have no response or even deterioration in end organ function $[7,8]$. The current cost of CFTR modulators is >US\$270000 per patient, per year, so clearly the best outcome is required in view of the expense of treatment [9]. In the current era of precision medicine, patient-derived mini-organs (organoids) have emerged as an aide to provide personalised medicine to patients by predicting an individual's response to drugs.

Various cell models exist to replicate the multi-organ nature of CF disease. Each has its own advantages and disadvantages $[10,11]$. To create an organoid, biopsies are taken from organs most affected by CF disease, the airways and gut. Stem cells are then isolated and a miniaturised organ created in the laboratory. This organoid acts as a surrogate or an "avatar" for that particular patient [12]. As the organoid has been created from an individual's own cells, it closely replicates their cellular response to medications $[13,14]$. Organoids have a vast expansion capacity and can be frozen and re-tested to allow each patient to be tested for a variety of treatments.

To date, CF organoids have been applied in basic and translational research, including disease modelling and drug development $[12,15]$. CFTR modulators were discovered using high-throughput screening of compounds on recombinant cell lines and primary cultures of CF bronchial cells [16]. Current evidence suggests that organoids have the potential to facilitate selection of the most optimal mutation-specific therapy $[13,14,17,18]$. The US Food and Drug Administration (FDA) changed the drug regulatory environment when they permitted the use of in vitro analysis to inform the listing of different modulators for less common CFTR mutations, opening the door to in vitro testing becoming a driver in precision medicine. However, translation of organoids to a mainstream companion diagnostic test in CF requires further research to better define the specificity and sensitivity of the drug test results [19]. The first placebo-controlled double-blind trial (HIT-CF CHOICES) to incorporate organoid technology was announced in February 2020 [20]. This trial involves patients with rare CFTR genotypes, which are currently ineligible for approved CFTR modulators.

Whilst ongoing work interrogates the technical aspects of organoid technology, there is increasing recognition of the importance of patient involvement in research studies and clinical trial design [21]. For new technologies to make the leap from bench to bedside successfully, we need to consider the consumer's perspective $[22,23]$. Organoid technology as a companion diagnostic may be less acceptable to patients due to financial or ethical concerns about the future use of their organoids. These concerns may include the potential use of organoids in commercial ventures or in a way that does not fit with individual values [24]. Some of the limitations of organoids may influence a person's assessment of the acceptability. Limitations include the need for a repeat procedure to obtain a sample in the event of cell culture failure and the time taken to establish the cell cultures, which can be up to a few months, meaning that answers are not made available immediately [25].

Given a key requirement for adopting new technology is stakeholder acceptance, together with paucity of data in the CF community, we explored the acceptability of organoid technology in Australia.

\section{Methods and analysis}

Our objective was to assess the acceptability of using organoids as a companion diagnostic in individuals affected by CF and community comparisons. We hypothesised that people with CF, their parents or carers and community comparisons would find the use of organoid technology acceptable in this situation.

\section{Study design}

A cross-sectional observational design was used to survey individuals affected by CF and community comparisons. An online explainer video (figure 1 and supplementary video) was created to describe how personalised organoid technology might inform drug treatment decisions. Considering the similarities between a platform that uses patient-derived organoids and mouse-derived xenografts, this study protocol was based on that of WAKEFIELD et al. [25, 26] who investigated the acceptability of mouse-derived xenografts used in childhood cancer. We assessed acceptability over five domains. 
1) Willingness to use: Using a scale of one to seven, participants were asked to rate the likelihood that they would use organoid technology if it were offered to them. Participants were asked this question before and after being asked to rate seven potential advantages and seven potential disadvantages, to assess if changes occurred after deliberation.

2) Perceived advantages and disadvantages of organoids (supplementary table S1): On a scale of one "not at all important", to seven "very important", participants were asked to score seven possible advantages and seven possible disadvantages of organoids. These were adapted from WAKEFIELD et al. [25] to be more specific to CF treatment using our experience of managing patients with $\mathrm{CF}$.

3) Maximum acceptable cost: We asked participants the maximum out-of-pocket amount that they would be willing to pay for organoid technology. We provided several value ranges to select from, on a scale ranging from $\mathrm{A} \$ 20$ (€12/US\$13) to A $\$ 10000$ (€6000/US\$6500).

4) Maximum turnaround time: We asked participants to report the maximum time they would be willing to wait to receive results. Four options were provided: 2 weeks, 1 month, 3 months or 6 months.

5) Acceptable source of tissue: Participants were asked if the invasiveness of the procedure to obtain the sample would affect their decision. Response options of yes, no, maybe were provided. Participants were asked which sample site they would prefer and were able to select multiple options from those provided: nose, lung and/or rectum. A brief description of the procedure required to obtain each sample type was provided in the question text.

\section{Ethics approval}

The Sydney Children's Hospital (SCH) Network Human Research Ethics Committee approved this study, LNR/18/SCHN/526. Participants completed an electronic consent form prior to completing the questionnaire.

a)

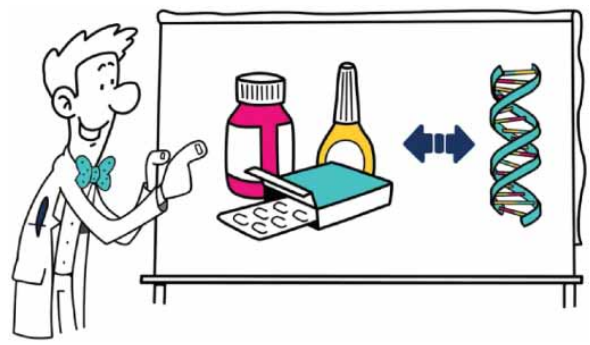

c)

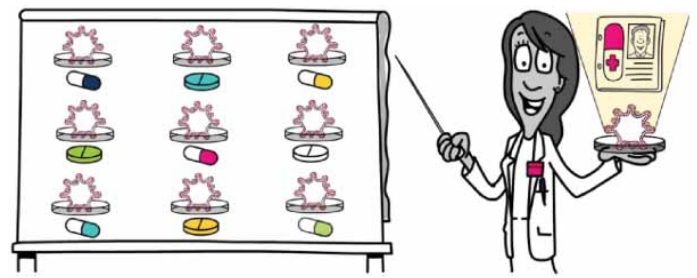

b)

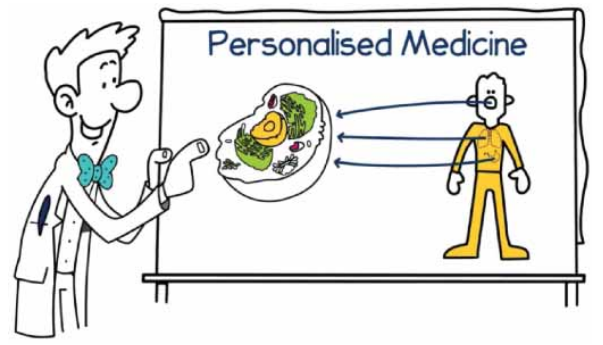

d)

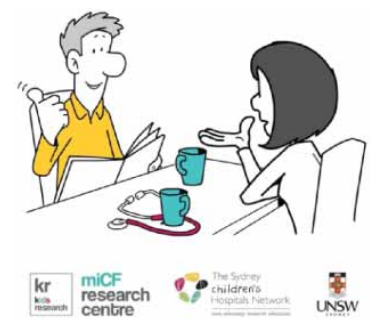

FIGURE 1 (a-d) Screen shots from the avatar acceptability explainer video. A 1.2-min video was used to describe personalised medicine to potential participants. A link to a questionnaire with 25 questions was placed below the video with a written invitation to take part in the study. The following script was used as the video's voiceover: "Only you are you, and your genes are unique to you. When it comes to medication, everyone responds to drugs differently. To put it simply, one size does not fit all. The best practice is to match medications to one's genetics. To truly understand how a patient might react to a drug it is ideal to test the drugs in a created environment that best reflects the patient. This is called personalised medicine. A way to find the perfect fit is by testing medications on cells collected from a person. The collected cells can be from the inside of a patient's own nose, lungs or stomach. The cells are grown in a special gel-like substance, which allows the cells to form mini organ-like structures. These mini-organs or organoids act like an 'avatar' for a person as they can be copied in the lab, so that millions of copies are available for different drugs to be tested on. Scientists can then potentially determine what works on a patient's avatar and from there suggest the best therapy for that specific patient. This approach would hopefully improve patient care and take the guesswork out of the game." The video remains live on the Sydney Children's Hospital YouTube page (https:// youtu.be/u24ldrzbJQw). 


\section{Participants}

We recruited four groups:

1) Adults with $\mathrm{CF}$ who were 18 years of age or older (CF adults).

2) Parents of children who were aged $<18$ years and diagnosed with CF (CF parents).

3) Adult community comparisons, aged 18 years and older, without a history of CF and no children under 18 years (non-CF adults).

4) Parent community comparisons, who had at least one child aged under 18 years, with no history of $\mathrm{CF}$ in either the parent or their child(ren) (non-CF parents).

Demographic details including age group, status as a parent of a child under 18, CF status and their child's CF status when applicable were collected. Participants were asked whether they had heard of the organoid technology before and if so, to rate the information they had heard as positive or negative on a five-point scale.

\section{Exclusions}

Any participant who was unable to read and understand English and any participant who answered that they did not understand the explainer video was excluded.

\section{Recruitment}

From February 2019 until January 2020, participants were invited to partake in the study via e-mail and posters displayed in CF clinics plus online media notices posted on social media platforms of Sydney Children's Hospitals Network (SCHN), CF Australia, Molecular and Integrative CF Research Centre (miCF $\mathrm{RC}$ ) and Cure $4 \mathrm{CF}$. Advertising material included a web address and QR code that linked to the study webpage hosted on the SCHN website.

\section{Data collection}

Participants were invited to watch an explainer video (figure 1). Participants who did not understand the video were invited to re-watch it or contact the study team. They were excluded if they continued to answer "not at all" when asked how well they understood the information in the explainer video. The online questions were not visible to these participants. The online format was used to ensure uniformity in the explanation of organoid technology and to reach a larger representative sample. Duplicate responses (indicated by IP address, e-mail address and survey demographic data) were removed.

\section{Data analysis}

A decisional balance ratio was calculated for each individual by dividing the participant's mean advantages ratings by their mean disadvantages ratings [27]. A ratio greater than one indicates that the participant's perceived advantages outweighed the perceived disadvantages and the organoid technology is acceptable. Likewise, ratios less than one indicate the technology is unacceptable. A value of one represented "decisional equivalence". In our data analysis, decisional equivalence results were grouped into the "not acceptable" category.

Data were analysed in Graphpad Prism Version 8. Paired data (pre- and post-questionnaire scores) were compared using a paired $t$-test. ANOVA and Chi-squared tests were used to compare groups. Log rank tests were used to compare maximum turnaround time and willingness to pay.

\section{Sample size}

With a sample size of 94 participants from the Australian CF population of 3400 [28] the margin of error of a finite-population-corrected 95\% confidence interval for a binary proportion is no larger than $10 \%$. The study remained open for 1 year to allow recruitment of the required number of CF participants.

\section{Results}

At the conclusion of the study, the QR code and links to the video had been used 322 times. Two hundred and twenty-two participants commenced the questionnaire using the links. Of these, 189 participants completed the consent form and initial information. One participant reported that they did not understand the video and did not progress further, and 188 completed the questionnaire. CF adults estimated mean reported age was younger than the other groups (figure $2 \mathrm{a}$ and supplementary figure S1). CF participants were more likely to have heard of the technology before (figure 2b). The majority (90 $(92 \%)$ ) of participants who had heard about the technology before had heard positive information (figure $2 \mathrm{c}$ ).

\section{Participants' willingness to use organoids remained high after considering the advantages and} disadvantages

All groups rated their willingness to use organoids highly prior to considering the advantages and disadvantages (mean score $>6.59$ out of 7 ) (figure $3 \mathrm{a}$ ). The mean score dropped within all groups once 
a)

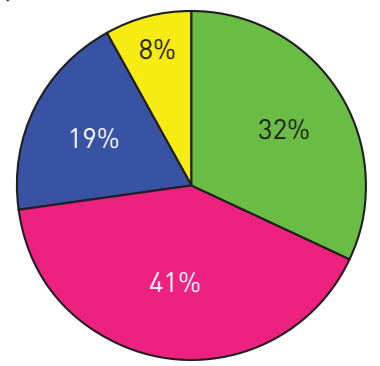

d)

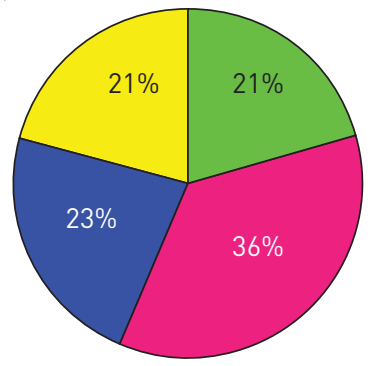

g)
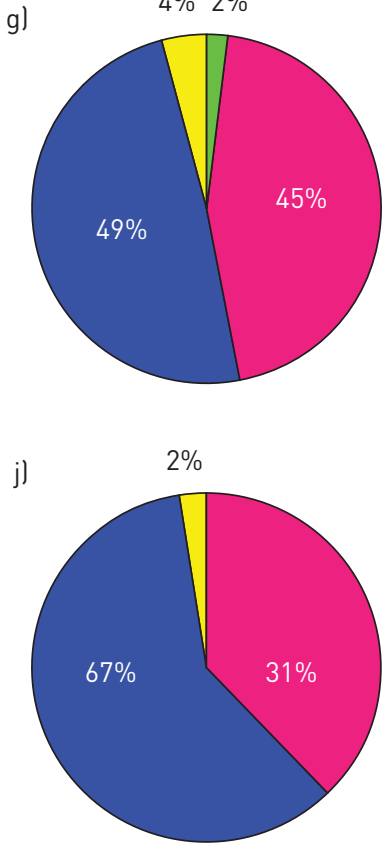

$18-25$ years

25-40 years

40-55 years

$\geqslant 55$ years b)

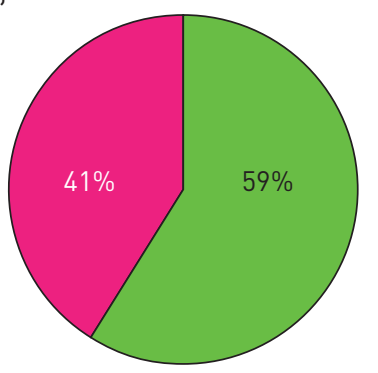

e)

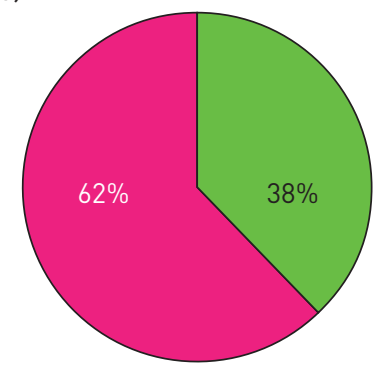

h)

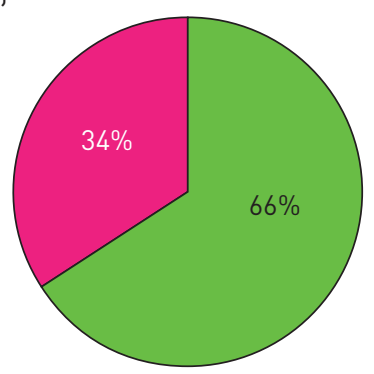

k)

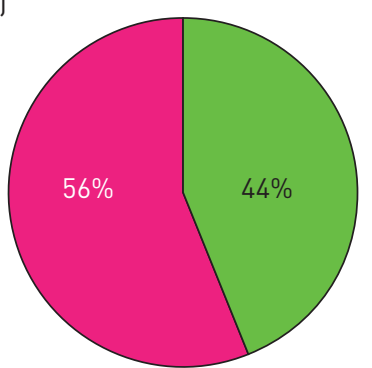

c)

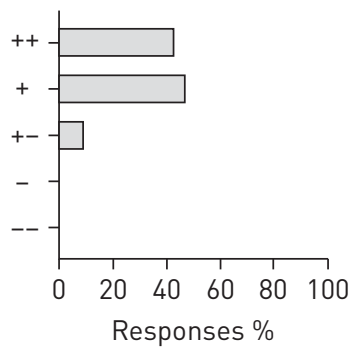

f)

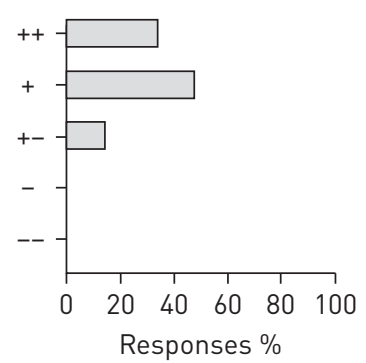

i)

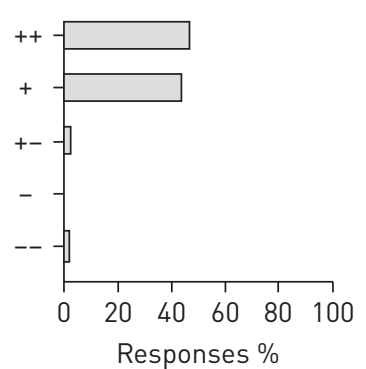

l)

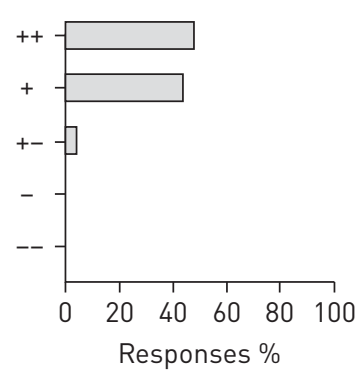

FIGURE 2 Participant groups and demographics. After watching the explainer video and giving consent to take part in the study, participants completed a set of questions about themselves to allow categorisation of their responses into four cohorts: a-c) cystic fibrosis (CF) adult (n=37), $d-f$ ) non-CF adult ( $n=53), g-i)$ CF parent $(n=53)$ or $j-()$ non-CF parent $(n=45)$. Data is displayed as a percentage of each response per group. 11 out of $37 \mathrm{CF}$ adults were also parents of children under 18 years. Their responses are only included in the CF adult group. a, d, g and j) Age groups of participants. A Chi-squared test demonstrated statistically significant difference in age between the groups. As data was collected as categorical variables, multiple Chi-squared tests were used to compare the groups and Bonferroni correction applied. CF adults were statistically significantly younger than both CF and non-CF parent groups. Non-CF adults were statistically different from CF and non-CF parents, but there was no significant trend identified. Using Bonferroni correction, $p<0.008$ was considered significant. $b$, e $h$ and $k$ ) Percentage of participants that reported that they had heard about the organoid technology prior to completing this survey. c, $f, i$ and $l$ ) Participants who had heard of the technology previously rated the type of information they had heard as totally positive (++), positive (+), mixed (+-), negative (-) or totally negative $(--)$. There was no significant difference between the type of information each group of participants had heard previously. In order to perform a Chi-squared test the single response of negative was combined into the response "mixed" in the CF parent group. n: number of participants. 
they considered the potential advantages and disadvantages (supplementary table S1). However, the mean score remained above six.

The advantages of organoids scored significantly higher than the disadvantages

All groups scored the perceived advantages significantly higher than the disadvantages (figure $3 \mathrm{~b}$ ). A decisional balance score above one (indicating the participant found the use of the organoid technology acceptable) was observed in 98 (100\%) non-CF participants, 36 (97\%) CF adults and 50 (94\%) CF parents (figure $3 \mathrm{c}$ ). When the raw decisional balance scores were compared, no significant difference between the groups was identified (supplementary figure S2).

When contemplating the seven proposed advantages, the most important perceived advantage overall was that the organoids may improve treatment selection, therefore improving the patient's quality of life and life expectancy. However, when the groups were looked at independently, the CF and non-CF parents scored organoids might help doctors choose the right drug more quickly which may avoid having to try several other drugs before finding the best one highest (supplementary table S2).

When considering the seven proposed disadvantages, the disadvantage with the highest overall importance rating was that the treatment recommended from the organoid testing may be unavailable or too expensive. However, CF adults rated patient may be recommended a treatment that is different to the most common treatment used or that is not compatible with their existing treatments as an equally important disadvantage. Non-CF parents' disadvantages ratings differed from the group as a whole: they rated the recommendation of a treatment that is different to the most common and that it may take some time to get the results as equally important (supplementary table S2).

CF parents were willing to pay more than community controls

Two CF adult participants elected not to answer this question. Whilst there was no statistically significant difference in the amount each group was willing to pay overall, 27 (51\%) CF parents reported that they were willing to pay up to $\$ 5000$. Whereas $11(31 \%)$ CF adults, $16(30 \%)$ non-CF adults and $16(36 \%)$ non-CF parents were willing to pay up to $\$ 5000$ (figure $4 \mathrm{a}$ ).

\section{CF participants were willing to wait longer than community controls for results/recommendations} from organoids

$\mathrm{CF}$ adults and parents were willing to wait longer than non-CF adults and parents. Six (16\%) CF adults versus three (6\%) non-CF adults were willing to wait 6 months. Eight (15\%) CF parents versus one (2\%) non-CF parent were willing to wait 6 months (log rank test $\mathrm{p}=0.0030$ ) (figure $4 \mathrm{~b}$ ).

a)

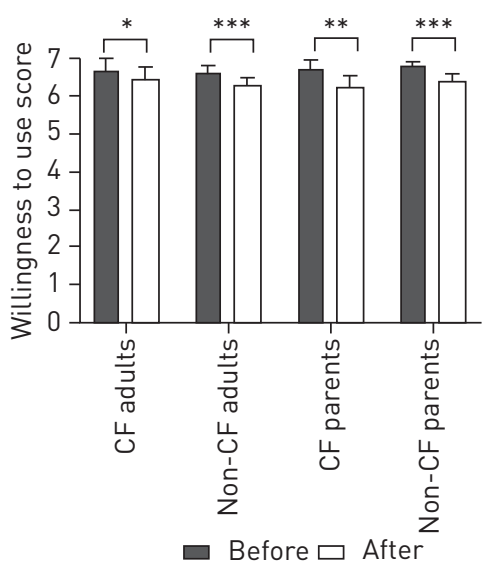

b)

\begin{tabular}{lccc}
\hline & $\begin{array}{c}\text { Advantages } \\
\text { mean } \pm \text { SD }\end{array}$ & $\begin{array}{c}\text { Disadvantages } \\
\text { mean } \pm \text { SD }\end{array}$ & p-value \\
\hline CF adults & $6.548 \pm 0.60$ & $3.131 \pm 1.37$ & $<0.0001$ \\
Non-CF adults & $6.458 \pm 0.59$ & $3.561 \pm 1.21$ & $<0.0001$ \\
CF parents & $6.598 \pm 0.60$ & $3.491 \pm 1.42$ & $<0.0001$ \\
Non-CF parents & $6.429 \pm 0.60$ & $3.602 \pm 1.14$ & $<0.0001$ \\
\hline
\end{tabular}

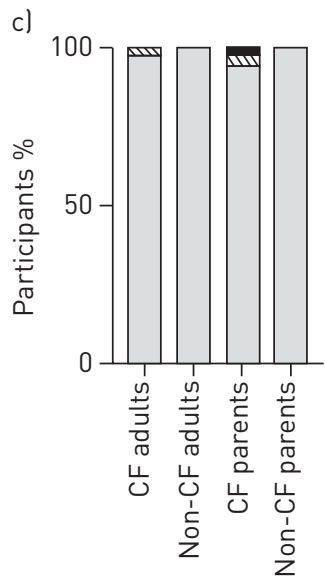

$\square$ Acceptable $>1$

- Equivalence $=1$

WI Not acceptable $<1$

FIGURE 3 Participants' assessment of the acceptability of organoid technology. a) Participants' willingness to use the organoid technology before and after considering potential advantages and disadvantages. The willingness to use scale provided ranged from one (unwilling) to seven (very willing to use). The mean willingness to use score is displayed by group. Willingness to use the organoid technology remained above a score of six in all groups. b) Participants scored each advantage or disadvantage on a scale from one (not at all important) to seven (very important). The mean advantages and disadvantages score for each group is displayed. c) Acceptability to use the technology was assessed by a decisional balance score, calculated for each participant by dividing the mean advantages score by the mean disadvantages score. Error bars represent standard deviations. CF: cystic fibrosis. ${ }^{*}: p<0.05 ;{ }^{* *}: p<0.01 ;{ }^{* * *}: p<0.001$. 
a)

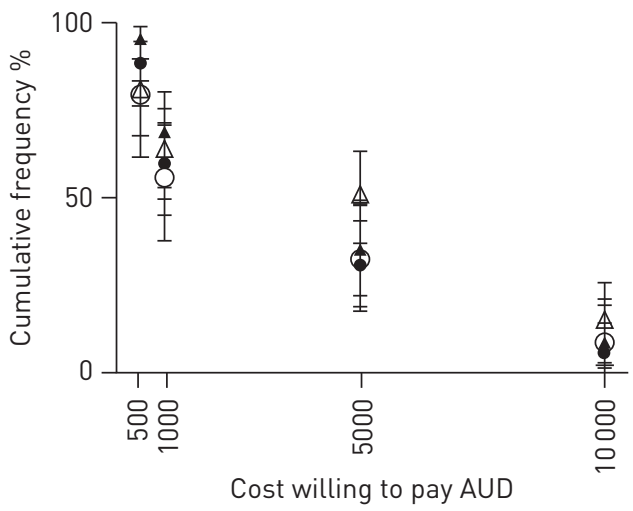

b)

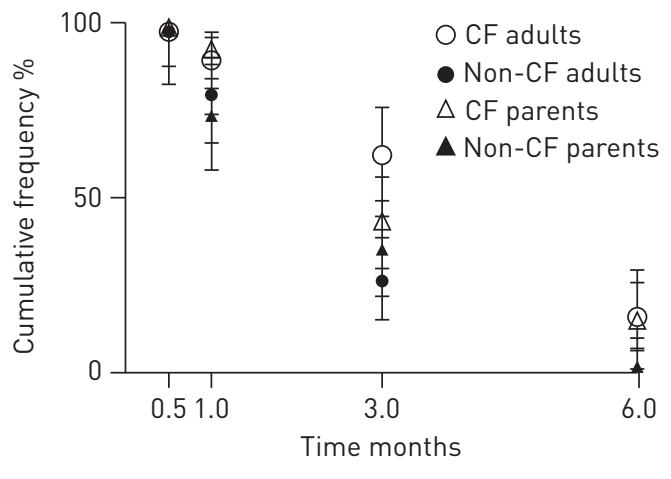

FIGURE 4 Participants' willingness to pay out of their own pocket and length of time they were willing to wait for the communication of test. a) Participants were asked to select from four Australian dollar value ranges between 500 and 10000 . The graph displays the proportion of participants willing to pay each out-of-pocket dollar amount to use the organoid technology. Increments represent the top value from each range. b) Participants were asked the maximum time that they were willing to wait to receive results from organoids. They were given the response options of 2 weeks, 1,3 and 6 months to select from. The graph displays the proportion of participants willing to wait each duration of time for the results of the organoid testing. Error bars represent $95 \%$ confidence intervals. CF: cystic fibrosis.

\section{Participants would choose the least invasive site of biopsy if given a choice of sites}

Most participants in each group indicated that the invasiveness of the biopsy to obtain the initial tissue sample would not affect their willingness to use the organoid technology (figure 5a). Most participants chose the nose $(175(93 \%))$ when they were also asked which site they would be more likely to choose if given a choice (figure 5b). CF parents were more likely to choose all three sites, 14 (26\%) CF parents compared to six (13\%) non-CF parents, four (11\%) CF adults and six (11\%) non-CF adults.

\section{Discussion}

Most participants found the use of organoids to guide treatment decisions for CF patients acceptable. A high willingness to use the technology was present amongst all groups surveyed, indicating that the technology is likely to be accepted by patients when organoids are implemented as a companion diagnostic. CF parents were willing to pay more than other groups surveyed, possibly reflecting the difference between considering a hypothetical illness compared with a real life-limiting disease that affects every aspect of day-to-day life. Both CF groups were willing to wait longer than non-CF groups for results, potentially reflecting their previous experience as chronic healthcare users, setting expectations of turnaround times. Most participants would choose the least invasive option for a biopsy site: the nose. However, over $10 \%$ of each group indicated a willingness to use all three biopsy sites, indicating the invasiveness of the procedure may not strongly impact their decision. CF parents were the most likely group to choose all three sites as acceptable options for biopsy for their child.

Key differences emerged when looking at the most important advantages and disadvantages for each participant group. Whilst overall the potential for organoids to improve therapy selection to improve patients' quality of life and life expectancy was the most important perceived advantage, parents rated the ability to choose the right drug more quickly without having to try several other drugs first as the most important. This may reflect parents' preference of experimenting on a cell model rather than their child.

This is the largest study of organoid acceptability amongst CF parents and adults, and it is the first with community comparisons. BoERs et al. [24] conducted 23 interviews with 14 adult CF patients and 12 parents of young CF patients in the Netherlands about organoid technology in CF. They conducted semi-structured qualitative interviews and elicited four themes. In general, their respondents were supportive of the technology, though they displayed an ambivalent attitude which was not seen in our online study; however, the methodology was quite different. Boers' qualitative approach allowed them to conduct exploratory research with a small group, gathering rich information on their perceptions of organoid technology without limiting the responses available to their participants. Our quantitative approach built upon information collected in previous studies to create a survey which could be administered to a larger number of people. This quantitative approach had the benefit of collating views from a larger group but was more limited in the response options available for participants.

Our study found very high acceptability ratings, much higher than those reported in WAKEFIELD et al.'s [26] study of 1550 Australasian cancer survivors and community controls. The cancer model involved the 


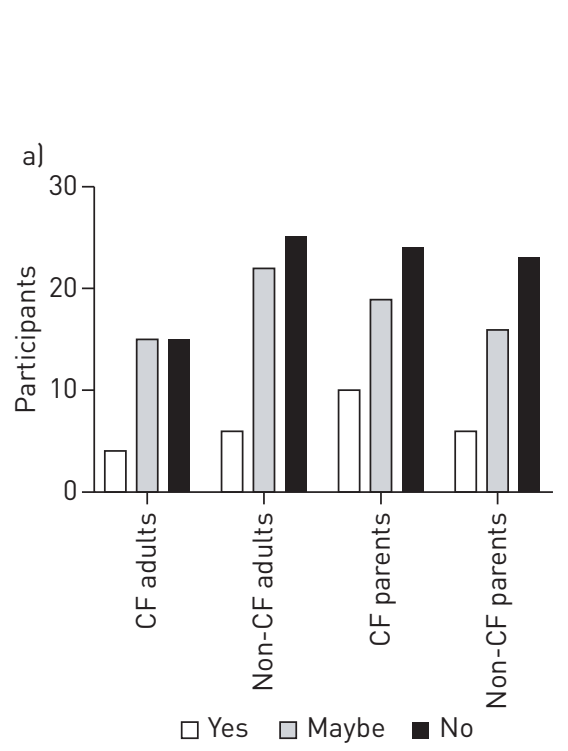

b)
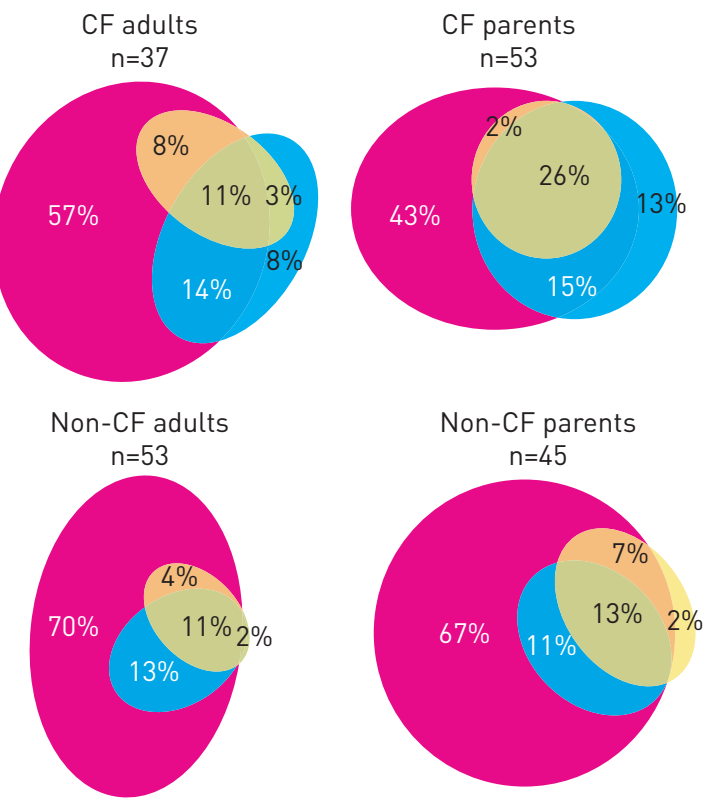

Nose Rectum Lung

FIGURE 5 Invasiveness of the biopsy effect on willingness to use organoids and participants' preference responses if given a choice of tissue biopsy site. a) Participants were asked if the invasiveness of the biopsy would affect their decision to use organoid technology. b) Site(s) of tissue biopsy chosen by participants when given a hypothetical choice to select one, two or three sites from the nose, lung and/or rectum. Data is displayed per participant group. The size of the ellipse section is proportional to the number of respondents. In all four cohorts, zero participants answered that they would only choose the lung or only the rectum, leading to completely overlapping sections for some response options. CF: cystic fibrosis.

use and sacrifice of mice. This may explain the 30\% lower level of acceptability amongst the community control groups in this study. Interestingly, however, the harming of animals was rated the least important disadvantage by participants [26]. In both our study and Wakefield's study, participants rated their willingness to use the technology lower once they had thought about the proposed seven advantages and disadvantages. This highlights the importance of discussion of the limitations of technology to ensure true informed consent is achieved before patients proceed with its use.

Participants indicated that they were willing to wait for the results of using organoid technology to guide CF therapy decisions, but not for too long. Nasal, lung and rectal organoids, which can be frozen and tested in the future, all require cell culture techniques to create them. Depending on the type of test performed, results can take up to 2 months [29]. Rectal biopsy samples, prior to being created as organoids, can be tested on the sample collection day to provide limited data on response to one or two drugs. Results from these samples could be available within a week [10]. Participants were willing to pay for the technology; however, only the majority of CF parents were willing to pay $>\$ 1000$, with $51 \%$ willing to pay $\$ 5000$. Similarly, in WAKEFIELD et al.'s study [26], over $50 \%$ of parents of cancer survivors were willing to pay up to $\$ 10000$ whereas $<50 \%$ of all other groups were willing to pay $>\$ 1000$. This trend of parents being willing to pay more for their child's treatment than adults will pay for their own may reflect the prioritisation of children over adults more generally. Organoid technology remains expensive. Health economic analyses, including potential savings from being able to choose an effective drug sooner, would be required to persuade government agencies of the merits of implementing this technology into mainstream clinical care.

Unsurprisingly when participants were given a choice about what site they would choose for a biopsy to be taken, most indicated a preference for the nose. The nose provides a minimally invasive site to brush to collect nasal epithelial cells. In contrast, sampling lung tissue involves a bronchoscopy, whilst sampling the gut involves a rectal biopsy done during a sigmoidoscopy. Some participants ( $23 \%$ of CF parents) were happy for all three sites to be sampled. This may reflect CF parents' comfort levels with elective general anaesthetic for their children, as many CF clinics in Australia perform annual surveillance bronchoscopy.

Using organoids to provide personalised medicine to patients is not limited to cancer and CF. A number of mini organ models derived from patients' cells are being developed to aid drug discovery and for personalised medicine in other diseases [30]. The acceptability of using organoids is therefore potentially 
relevant to a much broader range of diseases, particularly rare diseases and those with a heterogeneous phenotype.

\section{Limitations}

Our study is limited by the online approach. Whilst we know that 90 CF participants represents around $2.5 \%$ of the Australian CF community, we are unable to determine an accurate overall response rate, as we do not know how many people chose not to use a link in the advertising material displayed on posters, websites and social media. Our community comparison group is likely to have been particularly interested in health research given the placement of the study advertising and therefore may have been more likely to have a positive opinion regarding the use of organoids, though we note that $<41 \%$ of the community controls had heard about the technology before. Minimal demographic data was collected, limiting deeper analysis of the subgroups within the study. Respondents' educational level and sex were not available. Participants' reported response may not reflect their actual response if they were faced with a CF diagnosis and given the opportunity to use organoids. The results of our survey reflect the participants' current views on the use of organoids, which may change with time as new research and information becomes available. CF patients were represented in this study by participants with CF over 18 years; however, younger CF patients were only represented by their parents.

\section{Conclusions}

Organoids are increasingly being used in research around the world and are on the cusp of being translated to clinical practice. Participants in this study were willing to pay a significant amount for the technology. When given a choice, participants were more likely to choose the least invasive site for a tissue sample, the nose. This study demonstrates that using organoid technology to guide treatment decisions is likely to be accepted by the CF community.

Acknowledgements: We thank the study participants for their contributions. We appreciate the assistance from Sydney Children's Hospital, Randwick's respiratory department, CF Australia and Cure4CF in distribution of advertising material, special thanks to Leanne Plush, Amanda Thompson and Rhonda Bell and Nettie Burke. Advice regarding the statistical analysis was provided by Mark Donoghoe from Stats Central, UNSW.

Authors contribution: S.A. Waters and C.E. Wakefield conceived the study. S.A. Waters, C.E. Wakefield and L.K Fawcett designed the study. L.K. Fawcett, S. Sivam, P.G. Middleton and P. Wark recruited the participants. L.K. Fawcett performed the formal data analysis. L.K. Fawcett and S.A. Waters wrote the original draft of the manuscript. L.K. Fawcett, S.A. Waters, C.E. Wakefield, A. Jaffe, S. Sivam, P.G. Middleton, P. Wark and J. Widger wrote, revised and edited the manuscript.

Conflict of interest: None declared.

Support statement: This work was supported by Sydney Children's Hospitals Foundation and UNSW Triple I research grants. L.K. Fawcett is supported by the Rotary Club of Sydney Cove/Sydney Children's Hospital Foundation and UNSW postgraduate award scholarships. S.A. Waters and C.E. Wakefield are supported by the National Health and Medical Research Council of Australia (APP1188987 and APP1143767 respectively). Funding information for this article has been deposited with the Crossref Funder Registry.

\section{References}

1 Zemanick ET, Accurso FJ. Entering the era of highly effective CFTR modulator therapy. Lancet 2019; 384: $1886-1888$.

2 Lopes-Pacheco M. CFTR modulators: the changing face of cystic fibrosis in the era of precision medicine. Front Pharmacol 2019; 10: 1662.

3 Middleton PG, Mall MA, Dřevínek P, et al. Elexacaftor-tezacaftor-ivacaftor for cystic fibrosis with a single Phe508del allele. N Engl J Med 2019; 381: 1809-1819.

$4 \quad$ Elborn JS. Cystic fibrosis. Lancet 2016; 388: 2519-2531.

5 Cutting GR, Castellani C, Corey M, et al. Clinical and Functional Translation of CFTR (CFTR2). Cftr2 website 2013 Date last accessed: April 25, 2020; Date last updated: June 12, 2020. http://cftr2.org.

6 Drumm ML, Ziady AG, Davis PB. Genetic variation and clinical heterogeneity in cystic fibrosis. Annu Rev Pathol 2012; 7: 267-282.

7 Saiman L, Mayer-Hamblett N, Campbell P, et al. Heterogeneity of treatment response to azithromycin in patients with cystic fibrosis. Am J Respir Crit Care Med 2005; 172: 1008-1012.

8 Boyle MP, Bell SC, Konstan MW, et al. A CFTR corrector (lumacaftor) and a CFTR potentiator (ivacaftor) for treatment of patients with cystic fibrosis who have a phe508del CFTR mutation: a phase 2 randomised controlled trial. Lancet Respir Med 2014; 2: 527-538.

9 Tice JA, Kuntz KM, Wherry K, et al. 2020. Modulator Treatments for Cystic Fibrosis: Effectiveness and Value; Evidence Report. Institute for Clinical and Economic Review, April 27, 2020. Date last accessed: June 21, 2020 Date last updated: April 27, 2020. https://icer-review.org/material/cystic-fibrosis-2evidence-report/.

10 Awatade NT, Wong SL, Hewson CK, et al. Human primary epithelial cell models: promising tools in the era of cystic fibrosis personalized medicine. Front Pharmacol 2018; 9: 1429.

11 de Poel E, Lefferts JW, Beekman JM. Intestinal organoids for Cystic Fibrosis research. J Cyst Fibros 2020; 19 Suppl. 1, S60-S64. 
12 de Winter-de Groot KM, Berkers G, Marck-van der Wilt REP, et al. Forskolin-induced swelling of intestinal organoids correlates with disease severity in adults with cystic fibrosis and homozygous F508del mutations. J Cyst Fibros 2020; 19: 614-619.

13 Berkers G, van Mourik P, Vonk AM, et al. Rectal organoids enable personalized treatment of cystic fibrosis. Cell Rep 2019; 26: 1701-1708.

14 Pranke IM, Hatton A, Simonin J, et al. Correction of CFTR function in nasal epithelial cells from cystic fibrosis patients predicts improvement of respiratory function by CFTR modulators. Sci Rep 2017; 7: 1-11.

15 Grassi L, Alfonsi R, Francescangeli F, et al. Organoids as a new model for improving regenerative medicine and cancer personalized therapy in renal diseases. Cell Death Dis 2019; 10: 1-15.

16 Van Goor F, Hadida S, Grootenhuis PDJ, et al. Rescue of CF airway epithelial cell function in vitro by a CFTR potentiator, VX-770. Proc Natl Acad Sci USA 2009; 106: 18825-18830.

17 Dekkers JF, Berkers G, Kruisselbrink E, et al. Characterizing responses to CFTR-modulating drugs using rectal organoids derived from subjects with cystic fibrosis. Sci Transl Med 2016; 8: 344 ra84.

18 Debley JS, Barrow KA, Rich LM, et al. Correlation between Ivacaftor-induced CFTR activation in airway epithelial cells and improved lung function: a proof-of-concept. Ann Am Thorac Soc 2020; 17: 1024-1027.

19 Therapeutic Goods Administration. IVD companion diagnostics - Guidance on regulatory requirements. Date last accessed: June 20, 2020. Date last updated: Feb 14, 2020. https://www.tga.gov.au/sites/default/files/ivd-companiondiagnostics.pdf.

20 Wexler M. 2020. Organoid-Based Personalized Medicine Trial Enrollment Complete. Date last accessed: June 20, 2020. Date last updated June 12, 2020. https://cysticfibrosisnewstoday.com/2020/02/28/european-organoid-basedtrial-enrollment-complete/.

21 Symons T, McKenzie A, Straiton N. Australian Clinical Trials Alliance Toolkit. Date last accessed: April 25, 2020. Date last updated: June 12, 2020. https://involvementtoolkit.clinicaltrialsalliance.org.au/toolkit/understanding/whyinvolve-consumers/.

22 Jaffé A, Prasad SA, Larcher V, et al. Gene therapy for children with cystic fibrosis - who has the right to choose? $J$ Med Ethics 2006; 32: 361-364.

23 Delhove J, Osenk I, Prichard I, et al. Public acceptability of gene therapy and gene editing for human use: a systematic review. Hum Gene Ther 2020; 31: 20-46.

24 Boers SN, de Winter-de Groot KM, Noordhoek J, et al. Mini-guts in a dish: perspectives of adult cystic fibrosis (CF) patients and parents of young CF patients on organoid technology. J Cyst Fibros 2018; 17: 407-415.

25 Wakefield CE, Doolan EL, Fardell JE, et al. Protocol for the avatar acceptability study: a multiperspective cross-sectional study evaluating the acceptability of using patient-derived xenografts to guide personalised cancer care in Australia and New Zealand. BMJ Open 2018; 8: e024064.

26 Wakefield CE, Doolan EL, Fardell JE, et al. The Avatar Acceptability Study: survivor, parent and community willingness to use patient-derived xenografts to personalize cancer care. EBioMedicine 2018; 37: 205-213.

27 Tercyak KP, Hensley Alford S, Emmons KM, et al. Parents' attitudes toward pediatric genetic testing for common disease risk. Pediatrics 2011; 127: e1288-e1295.

28 Ruseckaite R, Ahern S, Ranger T, et al. The Australian Cystic Fibrosis Data Registry Annual Report, 2017. Monash University, Department of Epidemiology and preventative medicine 2019; 20: 1-45.

29 Gentzsch M, Boyles SE, Cheluvaraju C, et al. Pharmacological rescue of conditionally reprogrammed cystic fibrosis bronchial epithelial cells. Am J Respir Cell Mol Biol 2017; 56: 568-574.

30 Takahashi T. Organoids for drug discovery and personalized medicine. Annu Rev Pharmacol Toxicol 2019; 59: $447-462$. 